\section{Biokraftstoffe aus Sicht der Automobilindustrie}

\author{
von Frank Seyfried, Volkswagen AG
}

Eine der Herausforderungen zur Sicherung der zukünftigen Mobilität besteht in der Bereitstellung erneuerbarer Kraftstoffe für den Verkehrsbereich. Hierbei ist zu bedenken, dass diese Kraftstoffe zumeist eine völlig andere molekulare Struktur besitzen als fossile Kraftstoffe und damit auch gänzlich andere Eigenschaften. Dies erfordert in den meisten Fällen nicht nur eine neue Motorentechnologie sondern auch eine neue Produktions- und Verteilungsinfrastruktur. Dem entgegen ist es aus technologischen und ökonomischen Gründen nicht zielführend, neben Benzin und Diesel nun auch für die anderen in der Diskussion befindlichen Kraftstoffe (wie z. B. Biodiesel, Ethanol, Methanol, Erdgas, Flüssiggas, Dimethylether und Wasserstoff) eine neue Infrastruktur aufzubauen. Eine Zumischung dieser Kraftstoffe ist im Rahmen der bestehenden Normen zu bevorzugen. Insgesamt sinnvoller ist die Nutzung eines variablen Herstellungsprozesses für Kraftstoffe, der in der Lage ist, aus nahezu allen Primärenergien einen hochwertigen Kraftstoff konstanter Qualität herzustellen. Er sollte bei Umgebungsbedingungen flüssig und den konventionellen Kraftstoffen sehr ähnlich sein: Bei synthetischem Kraftstoff aus Biomasse - auch BtL, SunDiesel oder SunFuel genannt - ist dies der Fall. SynFuel heißt er, wenn er aus alternativen fossilen Energieträgern (z. B. Erdgas) hergestellt wird. Unabhängig von der Herkunft eröffnet er neue Möglichkeiten für zukünftige Verbrennungsprozesse. Synthetische Kraftstoffe insbesondere aus Biomasse - bieten eine intelligente Lösung. Für das nächste Jahrzehnt werden größere Anteile erwartet.

\section{Einführung}

Unter Umweltgesichtspunkten wird die Entwicklung von Kraftfahrzeugen und ihren Antrieben durch ständig verschärfte Abgasstandards bestimmt, die auch für konventionelle Antriebe praktisch bei Null liegen. Gleichzeitig gewinnen Maßnahmen zur Reduktion von Kraftstoffverbrauch und $\mathrm{CO}_{2}$-Emissionen einen ständig wachsenden Einfluss auf die Optimierung von Fahrzeug- und Antriebskonzepten.
Darüber hinaus steigt der weltweite Energieverbrauch bei sich abzeichnender sinkender Verfügbarkeit von preiswerter Primärenergie insbesondere mit Blick auf Mineralöl. Dadurch wächst die Bedeutung der Entwicklung von Alternativlösungen gegenüber der Nutzung von mineralölstämmigem Kraftstoff, um die individuelle Mobilität gewährleisten zu können.

Einen konkreten Ansatz innerhalb Europas bildet dabei die Selbstverpflichtung der Automobilhersteller, bis 2008 die $\mathrm{CO}_{2}$-Emissionen nach dem Neuen Europäischen Fahrzyklus (NEFZ) - bezogen auf den Flottenbetrieb von Neufahrzeugen - auf $140 \mathrm{~g} / \mathrm{km}$ zu begrenzen (vgl. Abb. 1 nächste Seite). Dies entspricht einer Reduktion von $25 \%$ (bezogen auf das Jahr 1995) und soll in zwei Anpassungsschritten erreicht werden.

Die Schlussfolgerung aus den genannten Rahmenbedingungen zur $\mathrm{CO}_{2}$-Reduzierung kann ein dreigeteiltes Vorgehen sein, das sowohl kurz- bis mittelfristige Konzepte beinhaltet, aber auch Entwicklungen genügend Aufmerksamkeit schenkt, die einer langfristigen Aktivität bedürfen. Die drei Lösungsansätze sind:

- konsequente Verbesserung von Wirkungsgrad und Emissionsausstoß der Antriebe,

- Einbeziehung von alternativen Energiequellen in die Kraftstoffherstellung,

- Entwicklung von $\mathrm{CO}_{2}$-effizienten Pfaden bei der Fahrzeugnutzung.

Eine der Herausforderungen ist dabei die gleichzeitige Abstimmung von verbesserten bzw. neuartigen Kraftstoffen und Antrieben. Die genannten Lösungsansätze fließen in der Kraftstoffstrategie der Volkswagen AG zusammen (Volkswagen $A G, 2004)$, über deren Inhalt es inzwischen einen breiten Konsens unter den führenden europäischen Automobilherstellern gibt (vgl. Abb. 2 nächste Seite).

Diese Strategie beruht auf vier Stufen der Weiterentwicklung und Kombination von Kraftstoffen und Antriebskonzepten (Motoren), die sich nach und nach ergänzen. Wie in Abbildung 2 skizziert, werden auf der Seite der Antriebskonzepte die Entwicklungen - ausgehend von Turbolader-Direkteinspritzern - über Hybridkonzepte (Kombination von Verbrennungsund Elektromotor), kombinierte Verbrennungssysteme (Zusammenführung des Otto- und Die- 
Abb. 1: Vergleich der Selbstverpflichtung der europäischen Automobilhersteller (ACEA) zur $\mathrm{CO}_{2}$ Reduzierung mit der Emission der Neuwagen-Flotte

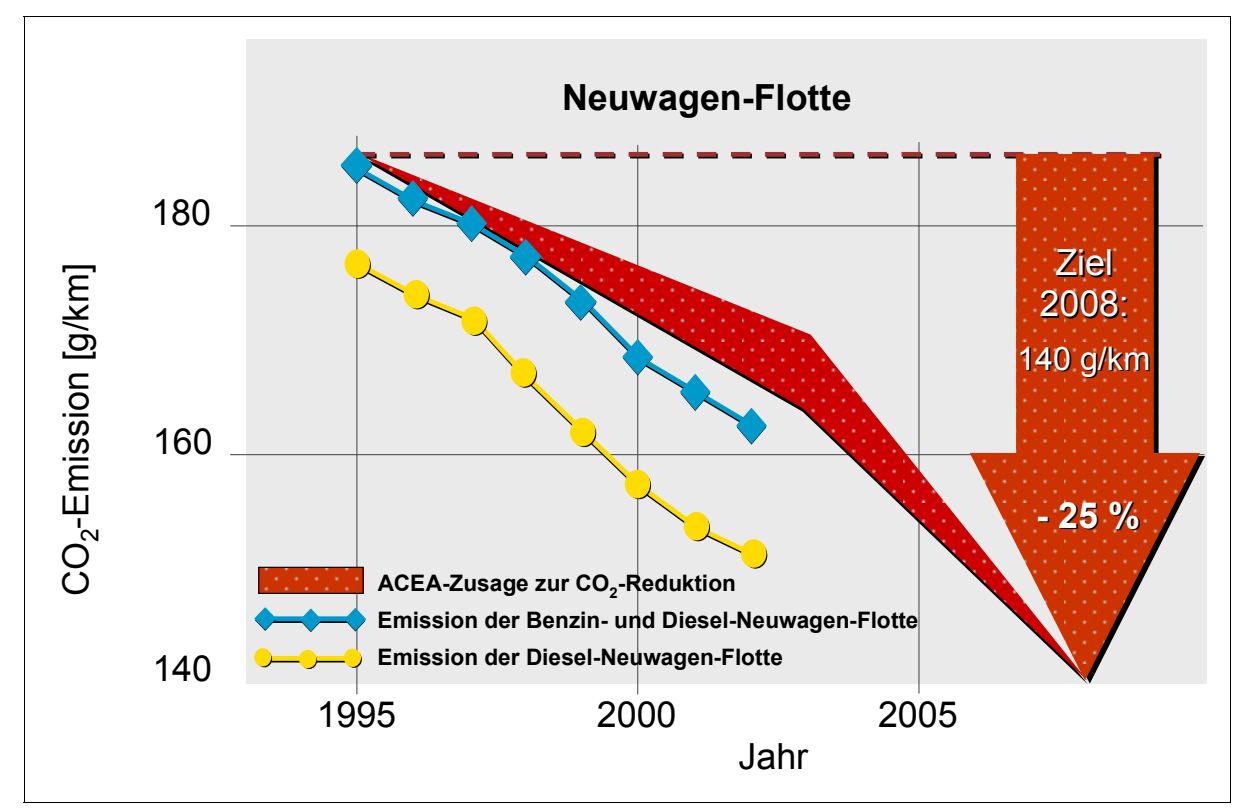

selmotors zu einem Konzept) bis hin zur Brennstoffzelle führen.

Parallel zu dieser Entwicklung müssen die entsprechenden Kraftstoffe bereitgestellt werden. Stufe 1 beinhaltet die Weiterentwicklung heutiger Kraftstoffe. In Stufe 2 werden synthetische Kraftstoffe (SynFuel) aus Erdgas (Gas-toLiquid, GtL) und ggf. aus Kohle (Coal-toLiquid, $\mathrm{CtL}$ ) - in Verbindung mit einer $\mathrm{CO}_{2-}$ Sequestrierung $^{1}$ - den Kraftstoffmarkt ergänzen. In Stufe 3 erfolgt der Wechsel von der fossil basierten Herstellung synthetischen Kraftstoffs zu nahezu $\mathrm{CO}_{2}$-neutral hergestelltem Synthesekraftstoff aus Biomasse (Biomass-to-Liquid, BtL), wie z. B. aus Holz, Stroh, Mais oder anderen angebauten Energiepflanzen. Stufe 4 bildet letztendlich die Phase der Wasserstoffwirtschaft aus erneuerbaren Energiequellen und dessen Nutzung in der Brennstoffzelle. Bis dahin sind jedoch noch wesentliche Technologiebarrieren bezüglich Speicherdichte im Fahrzeug, Infrastruktur oder Herstellungskosten zu beseitigen.

\section{Abb. 2: Kraftstoffstrategie der Volkswagen AG}

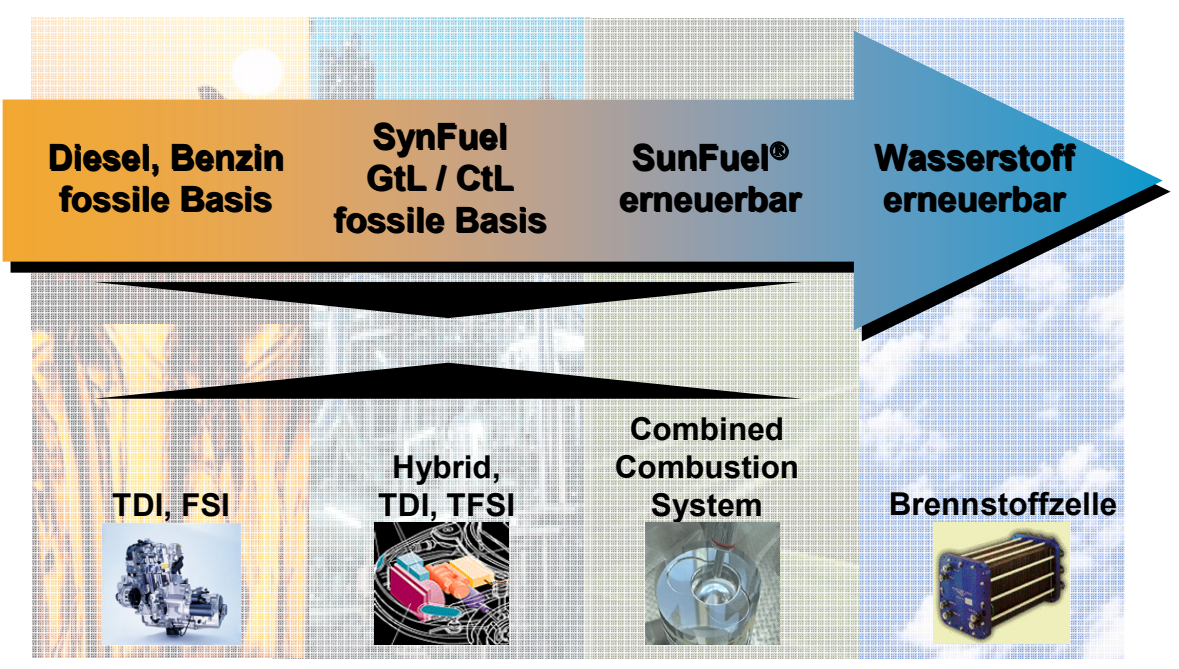


Im nachfolgenden Kapitel 2 werden zunächst die klassischen alternativen Kraftstoffe Biodiesel, Ethanol und Erdgas vorgestellt, gefolgt von der Bereitstellung von synthetischem Kraftstoff aus Erdgas (siehe Kap. 3). Der Schwerpunkt des Beitrags konzentriert sich auf die Bereitstellung und vergleichende Bewertung von synthetischem Kraftstoff aus Biomasse (siehe Kap. 4). Neben Potenzialabschätzungen werden hierzu Ergebnisse aus Lebenszyklusanalysen und Emissionsanalysen vorgestellt und mit herkömmlichen Kraftstoffen verglichen.

Die in diesen Kapiteln vorgestellten Ergebnisse resultieren aus eigenen Untersuchungen der Volkswagen AG bzw. aus Analysen, die in ihrem Auftrag durchgeführt wurden.

\section{Klassische Alternativen - Biodiesel, Ethanol und Erdgas}

\subsection{Biodiesel}

Unter dem Begriff Biodiesel wurde ursprünglich ein durch Methanol umgeestertes Rapsöl (Rapsölmethylester, RME) verstanden, dessen Eigenschaften durch die Veresterung des Pflanzenöls grundsätzlich verbessert worden sind. Inzwischen ist der Begriff Biodiesel aber erweitert worden und umfasst auch veresterte Fettsäuren (Pflanzenöle, Tierfette, gebrauchte Speiseöle). Der Marktanteil von Biodiesel beträgt in Deutschland derzeit 2 Vol.-\%. Nach einer flächendeckenden Versorgung des Marktes (z. B. EU 25) mit einem $5 \%$-Blend (Zumischung) ist die Steigerung des Biodieselgehaltes im Diesel auf zunächst bis zu 10 Vol.-\% später auch bis zu 20 Vol.-\% denkbar. Der Einsatz von reinem Biodiesel ist aus Emissionsgründen nicht zielführend und nicht notwendig, da das Beimischungspotenzial bei weitem nicht ausgeschöpft ist.

\subsection{Ethanol}

Als Beimischung zum Benzin wird Ethanol voraussichtlich in nahezu allen Märkten an Bedeutung gewinnen. Derzeit ist der Marktanteil in Europa mit unter $1 \%$ noch vernachlässigbar. Am günstigsten wird Ethanol nicht als reiner Kraftstoff sondern chemisch gewandelt als ETBE (Ethyltertiärbuthylether) eingesetzt. ETBE entsteht aus einer Reaktion von Ethanol (60\%) mit Isobuten (40\%). ETBE kann dem Benzin gemäß der europaweit gültigen Norm für Ottokraftstoff (EN 228) bis zu 15 Vol.-\% ohne kundenrelevante Auswirkungen zugemischt werden. Sollte eine ETBE-Produktion in den geforderten Mengen nicht möglich sein, kann Ethanol direkt nach der EN 228 bis zur Erfüllung einer Zumischung von 5 Vol.- $\%$ zum Benzin zugemischt werden. Die Erfüllung der EU-Biokraftstoffrichtlinie - bis 2010 soll ein energetischer Anteil der Biokraftstoffe von 5,75\% realisiert sein erfordert bezüglich der Ethanolbeimischung zum Benzin einen Mengenanteil von 8,7 Vol.$\%$, aufgrund des geringeren Energiegehalts von Ethanol. Nach der Erfüllung der flächendeckenden Zumischung von 5 Vol.-\% Ethanol ist in einem zweiten Schritt eine Zumischung von Ethanol bis zu 10 Vol.-\% denkbar, mit der dann die EU-Richtlinie erfüllt werden kann. In diesem Zeitraum könnte die EU-Kraftstoffnorm entsprechend geändert werden.

Alternativ wird die Einführung von Flexible-Fuel-Fahrzeugen stark diskutiert, die mit beliebiger Ethanolkonzentration im Benzin zwischen 0 (Reinbenzin) und 85 (E85) Vol.-\% betrieben werden können. Gegen diese Einführung sprechen sowohl volkswirtschaftliche als auch technische Gründe. Zum einen würde eine Marktdurchdringung (EU 25) von 10 Vol.-\% Produktionskapazitäten von ca. 13 Mio. t Ethanol pro Jahr benötigen. Dies ist eine Größenordung, die auch bei progressivem Vorgehen nur über viele Jahre hinweg aufgebaut werden kann. Darüber hinaus wären Investitionen in die Infrastruktur (z. B. Tankstellennetz) für die Einführung eines neuen Kraftstoffs kontraproduktiv. Im Gegensatz dazu würde in einem flächendeckenden Ansatz der erneuerbare Kraftstoff durch eine Beimischung zum herkömmlichen Kraftstoff von der gesamten Fahrzeugflotte genutzt und nicht nur von Neufahrzeugen. Außerdem treten bei hohen Ethanolkonzentrationen technische Schwierigkeiten wie Kaltstart- und Einspritzprobleme auf, die insbesondere in sparsamen, direkt einspritzenden Ottomotoren von Nachteil sind.

\subsection{Erdgas}

In den nächsten Jahren wird zunehmend Erdgas als Kraftstoff eingesetzt werden. Eine Ursache hierfür ist in Deutschland die Steuerreduzierung auf derzeit $155 € / \mathrm{t}$, gegenüber 
Benzin mit $855 € / \mathrm{t}$ und Diesel mit $578 € / \mathrm{t}$. An dieser Stelle sei angemerkt, dass sich die Höhe der Steuerreduzierung beim Erdgas durch dessen $\mathrm{CO}_{2}$-Vorteile von ca. 15 bis $20 \%$ gegenüber dem Benzin nicht rechtfertigen lässt. Erdgas wird direkt zum Fahrzeugantrieb insbesondere im Flottenbetrieb genutzt werden, allerdings ist kein Ersatz der heutigen Kraftstoffe durch Erdgas zu erwarten. Zu nachteilig sind die erhöhten Kosten für den Katalysator der Abgasnachbehandlung sowie die bekannten Platzprobleme durch die geringe Energiespeicherdichte von Erdgas.

\section{Synthetischer Kraftstoff aus Erdgas (Gas-to-Liquid)}

Die Herstellung synthetischer Kraftstoffe aus fossilen Kohlenwasserstoffen - insbesondere aus Erdgas - bietet in vielen Regionen der Erde, in denen Erdgas in entlegenen Quellen kostengünstig bereitgestellt wird oder als Erdölbegleitgas anfällt, beim heutigen Rohölpreis eine wirtschaftlich sehr interessante Alternative. Das aus dem Erdgas reformierte Synthesegas, eine Mischung aus Kohlenmonoxid und Wasserstoff, hat nahezu das ideale Mischungsverhältnis von ca. $1 \mathrm{~mol} \mathrm{CO}$ und $2 \mathrm{~mol}_{2}$ für eine anschließende Fischer-Tropsch-Synthese. Der aus der Fischer-Tropsch-Synthese gewonnene nahezu $100 \%$ ig paraffinische Fischer-TropschKraftstoff (FT-Kraftstoff) - vorzugsweise im Siedebereich von Diesel oder Kerosin - ist frei von Schwefel oder anderen mineralischen Verunreinigungen und vollkommen aromatenfrei (Gehalt an Aromaten: $<1 \mathrm{ppm}$ ). Firmen wie Shell und Sasol haben kürzlich Großinvestitionen in GtL-Produktionen beschlossen. Es wird etwa fünf bis sieben Jahre dauern, bis SynFuel aus Erdgas in größerem Umfang zur Kraftstoffversorgung beitragen kann.

Erstmals wurde das Emissionsverhalten des von Shell in Malaysia nach dem Verfahren der Shell Middle Distillate Synthese (SMDS) produzierten GtL-Kraftstoffs in einem Flottenversuch - an 25 Golf mit 1,91 TDI Aggregat $(74 \mathrm{~kW})$ - untersucht und mit konventionellem Dieselkraftstoff verglichen. Der Siedebereich dieses Kraftstoffs ähnelt dem von üblichem Diesel. Bereits ohne Anpassungen in der Motorsteuerung wurden deutliche Emissionsverbesserungen gegenüber konventionellem Diesel erzielt (vgl. Abb. 3). Die Emission an $\mathrm{NO}_{\mathrm{x}}$ und Partikeln $(<10 \mu \mathrm{m})$ reduzierten sich um 6 bzw. $26 \%$; die Emission an Kohlenwasserstoffen (HC) und CO wurden sogar um 63 bzw. $91 \%$ vermindert.

\section{Abb. 3: Vergleich der Emissionen im Flottentest bei Synthesediesel (GtL) mit konventionellem Diesel}

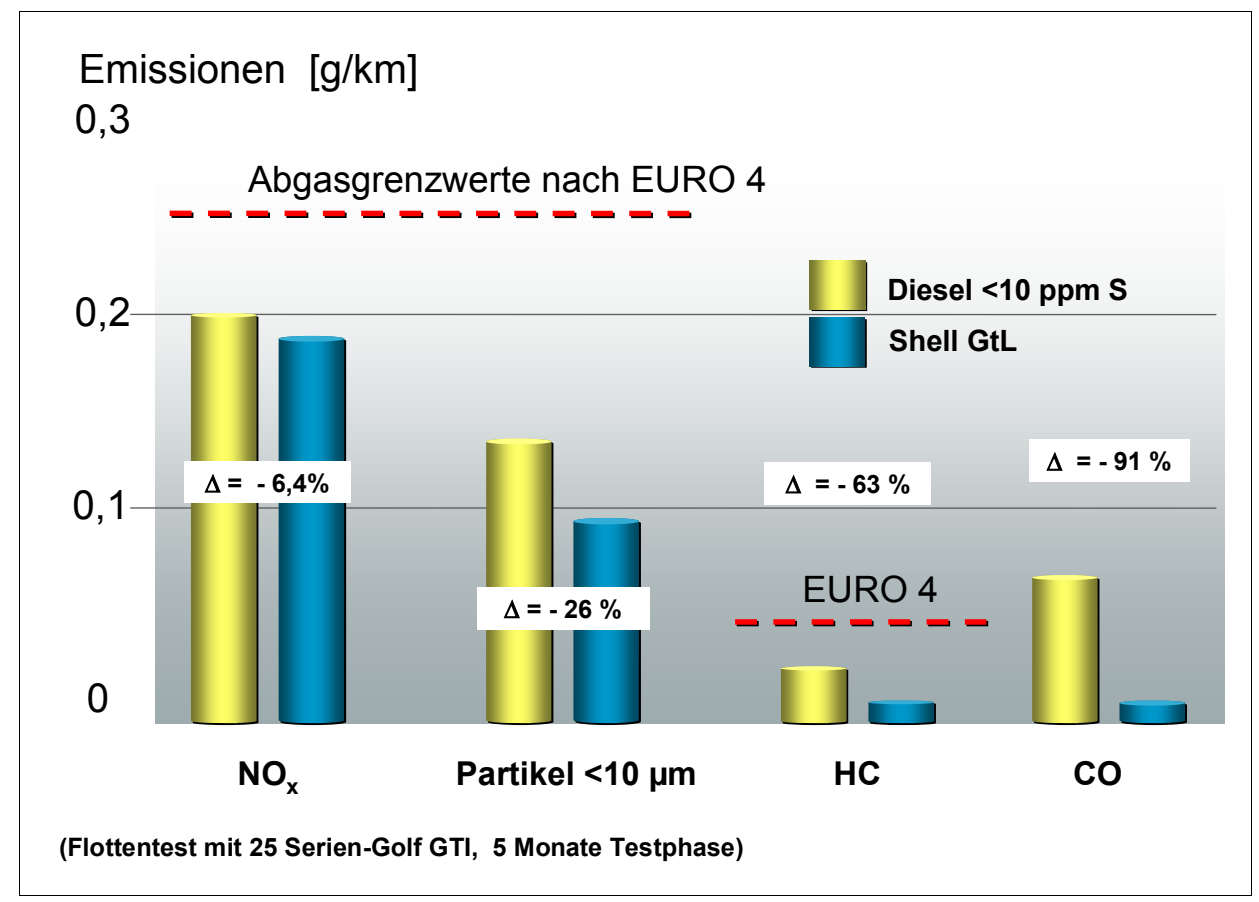


Der volumetrische Verbrauch blieb konstant, trotz der mit $0,78 \mathrm{~kg} / 1$ gegenüber konventionellem Diesel um $6 \%$ verringerten Dichte, die $\mathrm{CO}_{2}$-Emissionen sanken um $4 \%$. Der Shell GtL-Kraftstoff setzt sich aus etwas über $50 \%$ n-Paraffinen und über $45 \%$ isoParaffinen zusammen. Der Anteil von isoParaffinen ist für die Wintertauglichkeit unerlässlich, da dadurch der Cloudpunkt abgesenkt wird. Der Cloudpunkt entspricht der Temperatur, bei der durchgesaugter Dieselkraftstoff ein genormtes Filter blockiert. Iso-Paraffine senken die Cetanzahl - ein Maß für die Zündwilligkeit - gegenüber n-Paraffinen leicht. Dennoch erreicht der GtL-Diesel eine Cetanzahl von 73 (vgl. Tab. 1 in Kap. 4.3) mit den bekannten guten Verbrennungseigenschaften. Offensichtlich ist die Aromatenfreiheit in erster Linie ausschlaggebend für die deutliche Rußbzw. Partikelreduzierung (vgl. Abb. 3).

\section{Synthetischer Kraftstoff aus Biomasse (Biomass-to-Liquid)}

Die Erzeugung von Synthesegas als Zwischenprodukt zum Kraftstoff ermöglicht prinzipiell die Erzeugung eines praktisch identischen Synthesekraftstoffs aus jedem Kohlenwasserstoff. Beim Einsatz von Biomasse als Rohstoff wird die Kraftstoffnutzung in den natürlichen $\mathrm{CO}_{2}$ Kreislauf integriert und die $\mathrm{CO}_{2}$-Bilanz nahezu geschlossen. Abbildung 4 zeigt den mehrstufigen Biomass-to-Liquid-Prozess.
Biomasse hat in etwa ein molares $\mathrm{H} / \mathrm{C}$ Verhältnis von 1,6. Nach der Vergasung mit reinem Sauerstoff muss der Wasserstoffanteil im Synthesegas für die Fischer-Tropsch-Synthese erhöht werden. Großtechnisch erfolgt dies über die sog. Wassergas-Shift-Reaktion; CO wird dabei reduziert, bis ein Verhältnis von ca. $70 \%$ $\mathrm{H}_{2}$ und $30 \% \mathrm{CO}$ erreicht ist. In der nachfolgenden Fischer-Tropsch-Synthese entstehen Paraffine verschiedener Kettenlängen: das FischerTropsch-Rohprodukt. Dieses Rohprodukt wird dann in der anschließenden Wasserstoffnachbearbeitung in einer Isomerisierung oder einem Hydrocracking zum eigentlichen Kraftstoff aufgearbeitet. Nach der destillativen Trennung liegt der Hauptanteil des Kraftstoffs mit bis zu $80 \%$ im Siedebereich des Diesels vor.

\subsection{Lebenszyklusanalyse des BtL- Prozesses der Fa. Choren (SunFuel)}

In einer Lebenszyklusanalyse wurde der BtLProduktionsprozess der Fa. Choren aus Freiberg (Sachsen) zur Herstellung von „SunFuel“ auf der Basis von drei Szenarien untersucht. Das Szenario „Zukunft“ berücksichtigt, dass Wasserstoff, Sauerstoff und Elektrizität aus anderen erneuerbaren Quellen von außen eingebracht werden. Das Szenario ,autark“ bedeutet, dass alle Stoffe oder Energien, die innerhalb des Prozesses benötigt werden (wie Wasserstoff, Sauerstoff, Stickstoff und Elektrizität), innerhalb der Anlage erzeugt werden und keine

Abb. 4: Schematische Darstellung des Biomass-to-Liquid-Prozesses

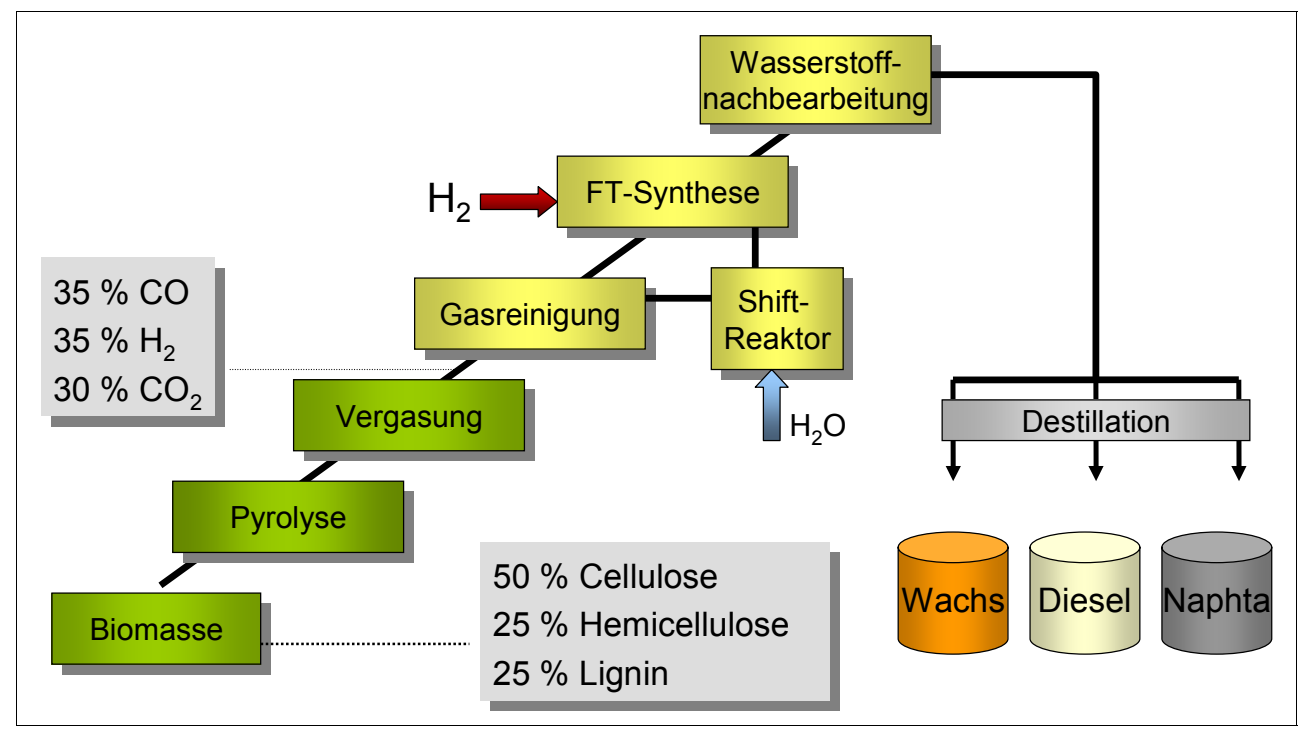




\section{Abb. 5: Reduzierung der Umweltbelastung durch SunFuel gegenüber konventionellem Diesel}

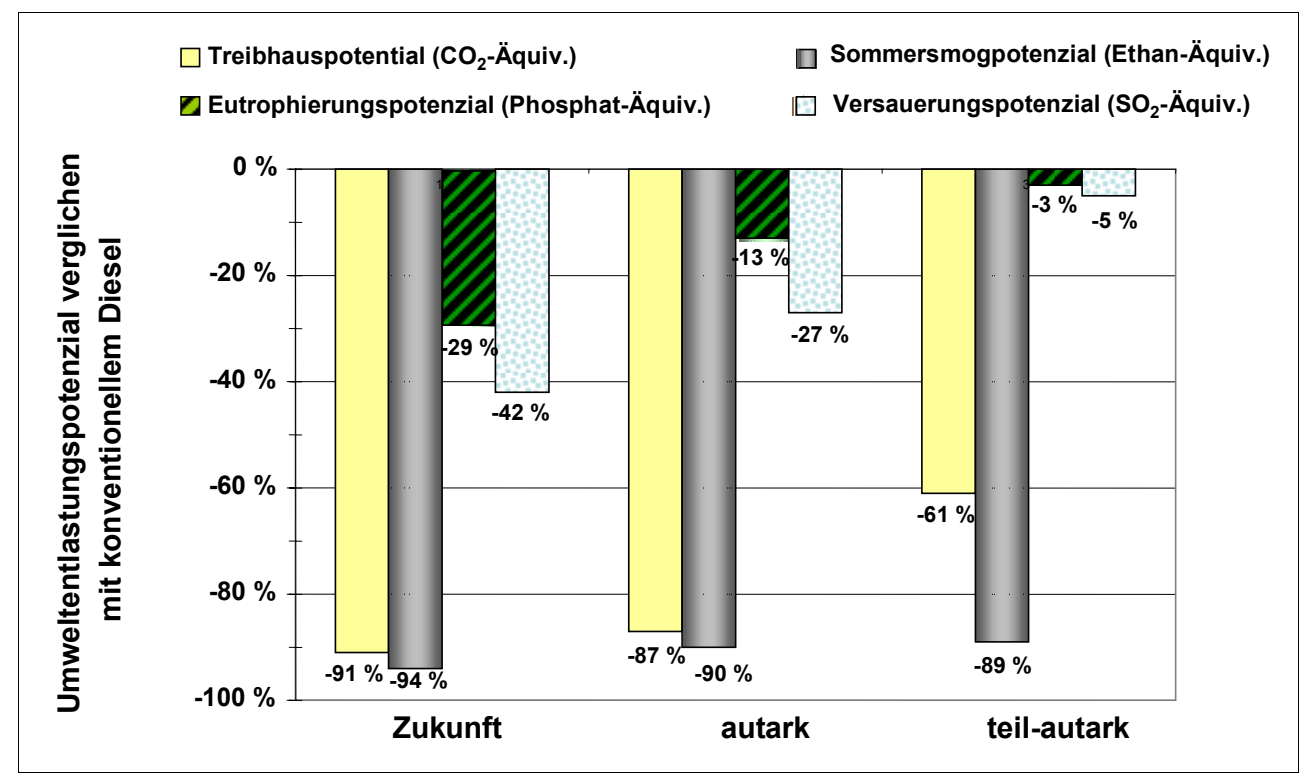

externen Energien zugeführt werden; in diesem Sinn kann von ,autarker Versorgung“ gesprochen werden. „Teil-autark" beschreibt den Fall, dass der benötigte Wasserstoff über einen Shift-Reaktor intern erzeugt, Sauerstoff und Elektrizität jedoch von außen bezogen werden. Abbildung 5 vergleicht die Ergebnisse für SunFuel und konventionellen Diesel im Hinblick auf Treibhausgaspotential, SommerSmog, Überdüngung (Eutrophierung) und Bodenversauerung.

Die Untersuchung zeigt die deutliche Verringerung der Umweltbelastungen durch SunFuel gegenüber konventionellem Diesel, insbesondere hinsichtlich Reduzierung der Treibhausgase und des Sommersmogs.

\subsection{Vergleich von SunFuel, Biodiesel und Ethanol hinsichtlich $\mathrm{CO}_{2}$-Reduzierung}

Eine wesentliche Größe bei der Entwicklung von Alternativen zur Mineralölnutzung ist die Verfügbarkeit des Kraftstoffs. Die derzeitige Biokraftstoff-Richtlinie der Europäischen Kommission fordert bis zum Jahr 2010 einen Marktanteil von 5,75\% durch biogene Kraftstoffe; für das Jahr 2012 wurde als Szenarienannahme ein Anteil von 6,25\% unterstellt.

Zur Realisierung dieses Zielwertes von $6,25 \%$ wurden drei Szenarien mit SunFuel, Biodiesel und Ethanol (EtOH) untersucht, die die Unterschiede der Biokraftstoffe bezüglich des $\mathrm{CO}_{2}$-Reduktionspotentials und der hierzu nötigen Anbaufläche verdeutlichen (siehe Abb. 6 nächste Seite). Den Analysen liegen Daten von heute ausgeführten Biodiesel- und Ethanolanlagen zugrunde. Bezüglich der Ethanolherstellung ist darauf hinzuweisen, dass sich Prozesse zur Ganzpflanzennutzung in der Entwicklung befinden, die ein deutlich verbessertes $\mathrm{CO}_{2}$-Reduktionspotential aufweisen werden. Die Aussage der Szenarienanalyse ist eindeutig: SunFuel bietet das höchste $\mathrm{CO}_{2}$-Reduktionspotential bei gleichzeitig geringster Inanspruchnahme von Anbaufläche. In Europa ist folglich die Nutzung von 15 Mio. ha Land zur Substitution von $20 \%$ des gesamten Kraftstoffs durchaus realisierbar. Neben den ökologischen Eigenschaften und der Verfügbarkeit ist natürlich auch das Emissionsverhalten der synthetischen Kraftstoffe (BtL- und GtL-Kraftstoffe) für deren Bewertung von entscheidender Bedeutung.

\subsection{Emissionen bei der Verwendung von BtL- und GtL-Kraftstoffen}

Ziel der Untersuchungen war es, das Potential von BtL- und GtL-Kraftstoffen zur Emissionsreduzierung herauszuarbeiten, im Vergleich zu konventionellem Diesel. Hierzu wurden zwei verschiedene Siedeschnitte von BtL-Kraftstoffen der Fa. Choren untersucht, der eine im Sie- 
Abb. 6: Szenarien zum $\mathrm{CO}_{2}$-Reduktionspotential und Flächenbedarf von SunFuel, Biodiesel und Ethanol in der EU

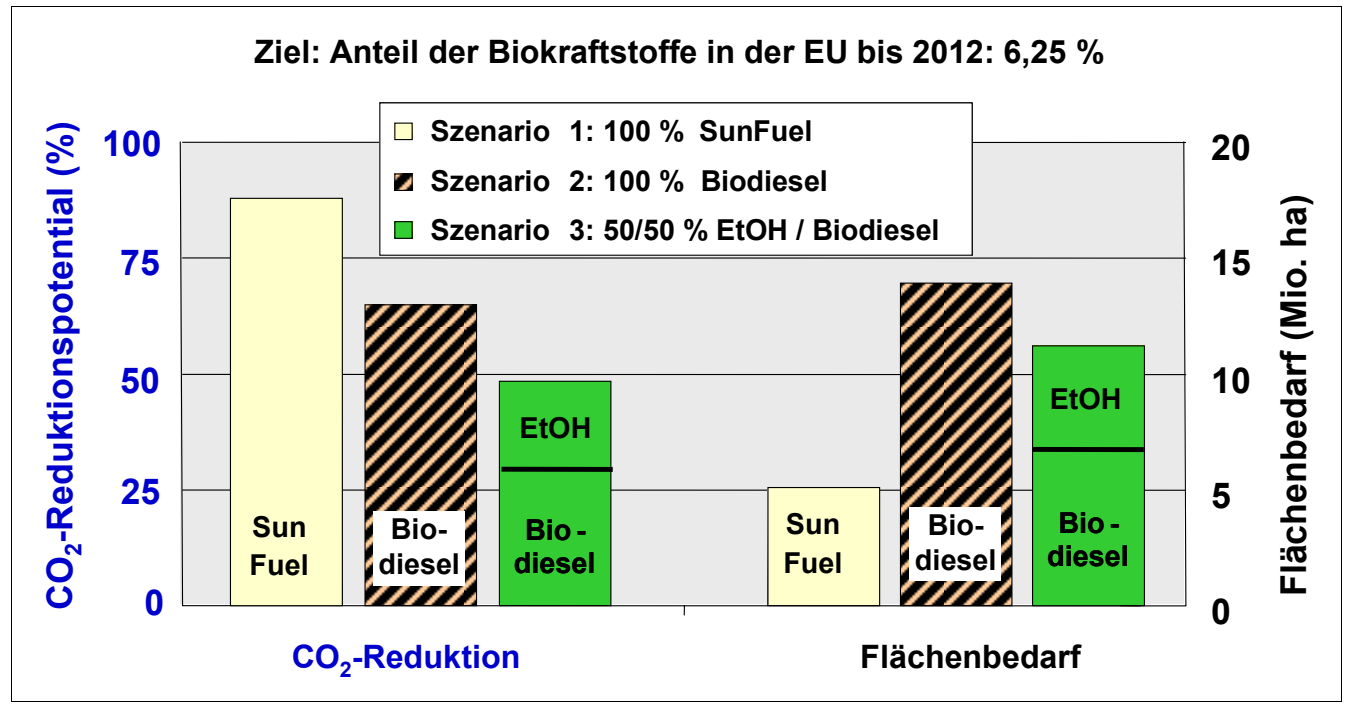

debereich von Kerosin der andere im Siedebereich eines Leichtdiesels. Als dritter FischerTropsch-Kraftstoff wurde GtL von Shell eingesetzt. Die Emissionsmessungen wurden an einem 2 l-4V-TDI Motor (103 kW) ohne jegliche konstruktive Änderung durchgeführt. In Tabelle 1 sind einige Eigenschaften der eingesetzten Kraftstoffe aufgelistet.

Aufgrund der Aromatenfreiheit ist die Dichte der FT-Kraftstoffe trotz des gleichen Siedebereiches niedriger als beim Referenzdiesel. Beim Heizwert fällt die Differenz mit $<1 \%$ geringer aus. Wesentlicher Unterschied zwi- schen den Synthesekraftstoffen ist in diesem Fall der Isomerisierungsgrad. Die beiden BtLKraftstoffe bestehen zu $89 \%$ bzw. $95 \%$ aus nParaffin, wogegen der GtL-Kraftstoff einen iso-Paraffinanteil von ca. $45 \%$ aufweist. Dies wird auch an der Cetanzahl ersichtlich, die durch die Isomerisierung abnimmt, aber noch deutlich über der des Referenzdiesels liegt.

Im Fokus der Untersuchungen standen Messungen zur Partikel- und $\mathrm{NO}_{\mathrm{x}}$-Emission. Dazu wurden die Abgasrückführrate und der Einspritzzeitpunkt für stationäre Zustände so variiert, dass zum einen ein hinsichtlich Partikel-

Tab. 1: Eigenschaften der GtL- und BtL-Kraftstoffe im Vergleich zu konventionellem Diesel

\begin{tabular}{|l|c|c|c|c|c|}
\hline & Einheit & $\begin{array}{c}\text { Konvent. Diesel } \\
\text { (Referenz) }\end{array}$ & $\begin{array}{c}\text { BtL- } \\
\text { Kerosin }\end{array}$ & $\begin{array}{c}\text { BtL- } \\
\text { Leichtdiesel }\end{array}$ & $\begin{array}{c}\text { GtL- } \\
\text { Diesel }\end{array}$ \\
\hline Dichte $\left(\right.$ bei $\left.15^{\circ} \mathrm{C}\right)$ & $\mathrm{kg} / \mathrm{m}^{3}$ & 0,83 & 0,76 & 0,77 & 0,78 \\
\hline \multicolumn{1}{|c|}{ Unterschied zur Referenz } & $\%$ & & $-9,4$ & $-7,2$ & -6 \\
\hline Viskosität $\left(40^{\circ} \mathrm{C}\right)$ & $\mathrm{cSt}$ & 2,8 & 1,5 & 2,3 & 3,5 \\
\hline Cetanzahl & & 53 & 79 & 94 & 73 \\
\hline Siedebeginn & ${ }^{\circ} \mathrm{C}$ & 170 & 179 & 212 & 241 \\
\hline Siedeende & ${ }^{\circ} \mathrm{C}$ & 370 & 276 & 326 & 355 \\
\hline Heizwert $\left(\mathrm{H}_{\mathrm{u}}\right)$ & $\mathrm{MJ} / 1$ & 36 & 33,2 & 33,8 & 34,1 \\
\hline H / C-Verhältnis & & 1,97 & 2,12 & 2,13 & 2,1 \\
\hline
\end{tabular}




\section{Abb. 7: Partikel- und $\mathrm{NO}_{\mathrm{x}}$-Emissionen von GtL- und BtL-Diesel in Relation zu konventionellem Diesel}

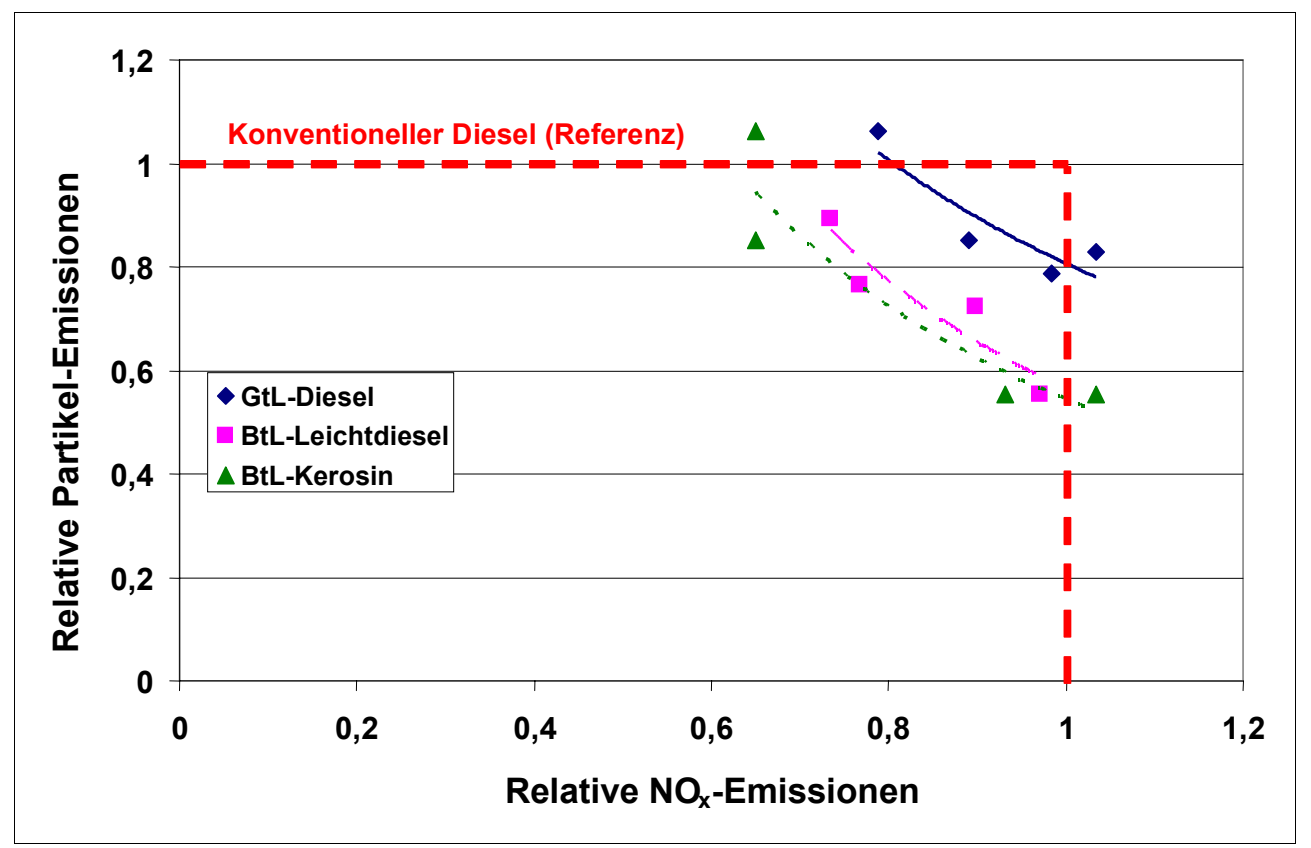

Emission und zum anderen ein hinsichtlich $\mathrm{NO}_{\mathrm{x}}$-Emission optimierter Zustand für den Neuen Europäischen Fahrzyklus (NEFZ) festgelegt werden konnten. Der NEFZ ist gesetzlich seit 1996 zur Ermittlung des Kraftstoffverbrauchs und der Abgasemissionen vorgeschrieben. Abbildung 7 zeigt die relativen Partikel- und $\mathrm{NO}_{x^{-}}$ Emissionen des GtL- bzw. BtL-Diesels im Vergleich mit konventionellem Diesel.

Die durchgeführten Untersuchungen zeigten bei den BtL-Kraftstoffen im Vergleich zum GtL-Diesel deutlich günstigere Emissionswerte. Ursache hierfür ist vermutlich eine Überlagerung der Eigenschaften aus niedrigem Siedeende und hoher Cetanzahl. Es zeigt sich, dass im Grenzfall ein Reduktionspotential von ca. $30 \%$ Prozent bei $\mathrm{NO}_{\mathrm{x}}$ und nahezu $50 \%$ Prozent beim Partikelausstoß möglich ist, verglichen mit konventionellem Diesel als Referenz. $\mathrm{Zu}$ berücksichtigen ist hierbei, dass nur eine stationäre und noch keine dynamische Anpassung der Motoren durchgeführt wurde.

Ergebnisse zum Verbrauch und $\mathrm{CO}_{2-}$ Ausstoß sind für die BtL-Kraftstoffe in Abbildung 8 dargestellt - auch in dieser Abbildung in Relation zu konventionellem Dieselkraftstoff. Für die Fraktion des BtL-Leichtdiesels wurde eine $7 \% \mathrm{CO}_{2}$-Reduzierung bei minimal verbessertem Verbrauch festgestellt. Für die
BtL-Kerosinfraktion wurde zwar ein Mehrverbrauch von $4 \%$ gemessen, dennoch aber eine Verringerung der $\mathrm{CO}_{2}$-Emissionen um $2 \%$ ermittelt. Hier zeigt sich ein nennenswertes Potential für eine weitere Reduzierung des $\mathrm{CO}_{2}$-Ausstoßes.

\section{Abb. 8: Verbrauch und $\mathrm{CO}_{2}$-Emission von BtL-Kraftstoffen im Vergleich zu kon- ventionellem Dieselkraftstoff}

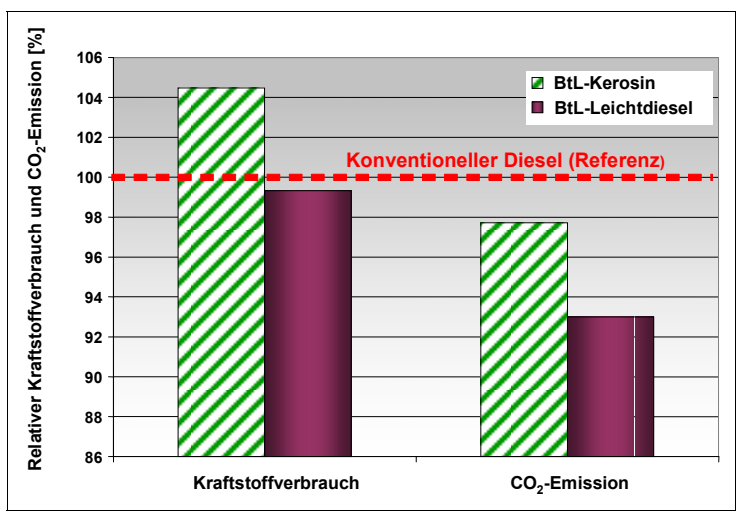

Noch deutlichere Vorteile der BtL-Kraftstoffe konnten beim Kaltstartverhalten bezüglich der Partikel-, $\mathrm{NO}_{\mathrm{x}}-$, HC-, $\mathrm{CO}-$ und $\mathrm{CO}_{2}$-Emissionen nachgewiesen werden. 
Insgesamt ist als Fazit festzuhalten, dass in der Einführung synthetischer Kraftstoffe, die spezifisch auf die Brennverfahren (Motoren) abgestimmt sind, ein beträchtliches Minderungspotential bei den Emissionen besteht.

\section{Anmerkung}

1) $\mathrm{CO}_{2}$-Sequestrierung bedeutet, dass das bei der Verbrennung (z. B. in einem Kohlekraftwerk) entstehende $\mathrm{CO}_{2}$ abgetrennt, verdichtet und anschließend in unterirdische Lagerstätten (z. B. Erdöl- / Erdgaslagerstätten, Kohleflöze) zur Endlagerung eingepresst wird.

\section{Literatur}

Volkswagen AG, 2004: Die Basis nachhaltiger Mobilität. Informationsbroschüre. Stand Mai 2004; http://www.volkswagen-nachhaltigkeit.de/nhk/nhk folder/de/download.Par.0019.Download.pdf; letzter Zugang: 14.03.2006

\section{Kontakt}

Dr. Frank Seyfried

Volkswagen AG

Konzernforschung, K-EFAK Kraftstoffe und Öle Brieffach 1778

38436 Wolfsburg

Tel.: +49 (0) 5361 / 936005

E-Mail: frank.seyfried@volkswagen.de

\section{Einordnung und Vergleich biogener Kraftstoffe - „Well-to-Wheel“-Betrachtungen}

\author{
von Jörg Schindler und Werner Weindorf, \\ Ludwig-Bölkow-Systemtechnik
}

Neben der Bereitstellung biogener Kraftstoffe wird in diesem Beitrag auch deren Verwendung im Fahrzeug untersucht. Diese ganzheitliche Vorgehensweise wird als Well-to-Wheel-Ansatz ${ }^{1}$ bezeichnet. Well-toWheel steht für die gesamte Prozesskette der Kraftstoffbereitstellung bis zur Verwendung im Fahrzeug. In einem ersten Schritt werden die jeweiligen Erzeugungspfade beschrieben und analysiert. Zunächst werden die Kosten der Kraftstoffherstellung und -verteilung bis zur Tankstelle dargestellt und bewertet. Daran schließt sich die Verwendung der Biokraftstoffe im Fahrzeug an. Die Darstellung stützt sich im Wesentlichen auf Studien, an denen die LudwigBölkow-Systemtechnik in den letzten Jahren beteiligt war. ${ }^{2}$

\section{Bereitstellungspfade für biogene Kraftstoffe}

Die verschiedenen Pfade, nach denen aus Biomasse Kraftstoffe hergestellt werden können, zeigt Abbildung 1 (nächste Seite). Die hierbei sich ergebenden Unterschiede in den Energiebilanzen und bei den Treibhausgasemissionen werden nachfolgend diskutiert.

Als einzelne Kraftstoffpfade werden berücksichtigt:

- Rapsölmethylester (RME) aus Rapsöl (über Umesterung),

- Methan aus dem Biogasreaktor (über anaerobe Vergärung),

- Ethanol aus Zuckerrüben, Mais, Weizen und schnellwachsenden Baumarten (über Fermentation / Vergärung),

- BtL-Kraftstoff (biomass to liquid) aus Restholz, Reststroh und schnellwachsenden Baumarten (über die Vergasung und Fischer-Tropsch-Synthese) und

- Wasserstoff, verdichtet oder verflüssigt, (über Vergasung von Restholz und schnellwachsende Baumarten). 